\title{
NATURE OF ERGASTIC SUBSTANCES IN SOME NIGERIAN ASTERACEAE
}

\author{
Omoigui, I.D. and O. S. A. Aromose
}

Department of Plant Biology and Biotechnology, University of Benin, PMB 1154, Benin City Correspondence author: ttiyanomoigui@yahoo.com

\begin{abstract}
Seeds of 110 species of the Nigerian Asteraceae were examined for fats and oils, proteins and starch using standard detection procedures. All the examined species except for 8 species were herbs. Fats and oils were found in all the species investigated comprising of both herbs and shrubs (100\% detection rate). Protein bodies were found in 23 species. While starch grains were found to be absent in all the investigated species. There was no correlation between life forms and the nature of ergastic substances in the family. The results from this study did not support the changes of names in the revision of the tribe Vernonieae as such this character cannot be used as a delimiting species.
\end{abstract}

Keywords: Ergastic substances, Shrubs, Herbs, Asteraceae.

INTRODUCTION

The Compositae (Asteraceae) family is nested high in the Angiosperm phylogeny in the Asterideae/Asterales. The family contains the largest number of described, accepted species of any plant family with 1600-1700 known genera distributed in over 24, 000 species found around the globe except for Antarctica (Funk, 2010). In Nigeria, the family is represented by 64 genera and 180 species (Adams, 1963).

The classification of the family Asteraceae is based on a combination of several specialized morphological characters e.g. capitula, highly reduced and modified florets, replacement of calyx by pappus, five epipetalous syngenecious anthers; bicarpellary condition; syncarpous inferior and unilocular ovary with single basal anatropus ovule, fruits as a rule are achenes (seeds).

Seeds are the primary storage organs of ergastic substances. Seeds and their byproducts are major components of the diet of man and its livestock. So far, man is dependent on a few cultivated plants (less than 30 plants) out of the thousands of plants. The knowledge of the stored products in the seeds of wild plants cannot be over emphasized. This is done with the view of harnessing the resources of these wild plants.

According to Erdtman (1962), ergastic substances are secondary products of plant metabolism which are formed at certain stages of their metabolic process and are retained when the taxon in question goes through further evolution while Gill and Idu (2001) are of the opinion that they are waste products resulting from cellular activities and are usually simpler in structure than their protoplasmic bodies.

Amongst the ergastic substances, starch occupies a unique position in the nutritional requirement of man. According to Gill et al., (1980) from the taxonomic point of view, starch grain characteristics are of great significance whereas protein bodies, fats and oils are of little taxonomic value as they occur sporadically in different taxonomic groups. They further suggested that the presence of tannins which is a primitive character often gets lost with increasing phylogenetic specialization. Gill et al., (1991) stated that the presence or absence of ergastic substances and their nature are of considerable importance in the evolutionary history of a taxon.

Using morphology, Robinson (1990) resurrected the genus Baccharoides and transferred 3 species of Vernonia to the genus. While Isawumi (1993) transferred 12 species from Vernonia to the same genus on the basis of the style base being surrounded by the nectar and involucral bracts with expanded foliose appendages which may be white or variously coloured. As a result, these have affected the Nigerian flora with change of name e.g. Vernonia gerberiformis $=$ Linzia gerberiformis, $V$. nigritiana $=L$. nigritiana, $V$. ituriensis var. occidental $=$ L. ituriensis var. occidental, V. migeodii = Vernoniastrum migeodii, $V$. bamendae = Orbivestus bamendae and $\mathrm{V}$. cinerea = Cyanthallium cinereum.

The importance of the nature of ergastic substances in plant systematics has been stressed by various workers (Omoigui and Gill, 1988; Idu and Gill, 1998)

The present study therefore is to evaluate if this character can aid in the classification of the members of the family.

\section{MATERIALS AND METHODS}

Seeds used in this investigation were collected in nature from different parts of Nigeria. Seed material was stored in $\left(27 \pm 2^{\circ} \mathrm{C}\right)$ in the laboratory before use. The method used for the determination of ergastic substances was outlined by Gill et al., (1980). Vouchers of the specimens studied were deposited in the herbarium of the University of Benin, Nigeria.

Seeds were put in petri dishes fitted with moist paper and left at room temperature for 12 hours to soften the seeds rapidly and the following tests were done. 


\section{Test for Starch}

Thin hard sections of the seeds were cut with sharp razor blades and placed on a slide. Few drops of Lugol's iodine solution were added. Starch if present reacted with iodine to give the characteristic blueblack colour.

\section{Test for Protein}

Seeds weighing $(0.5 \mathrm{~g})$ were macerated and $2 \mathrm{ml}$ of $60 \%$ of Nitric acid was added. Presence of protein was indicated by a white precipitate followed by a yellow solution on boiling.

\section{Test for Fats \& Oils}

Seeds weighing $(0.5 \mathrm{~g})$ were macerated with 2 drops of alcoholic Sudan III. Presence of fats and oils was indicated if on evaporation, the colour of Sudan III (which is red) was retained.

\section{RESULTS AND DISCUSSION}

In this study 110 species of Asteraceae distributed in 46 genera were studied. All the investigated species contained fats and oils (Table 1 ). This result agrees with the findings of Gill et al., (1984) and Gill and Idu (2001) concerning the occurrence of ergastic substances in different angiospermic families and their occurrence is sporadic and has no correlation with the habit of the plants. The presence of fats and oil in all the species in this study agrees with Omoigui and Gill (1988), Idu and Gill (1998) who worked on Southern Nigeria and West African Asteraceae respectively.

Protein was present in 23 species spread across different tribes of the family. They were found in Ceruana pratensis, Lactuca capensis, Launaea nana, L.rarifolia, Picris xylopoda, Sonchus oleraceus,
Anisopappus dalzelii, Porphryostemma chevalieri, Pulicaria crispa, P. undulata, Helichrysum foetidum, H. globosum, Emilia coccinea, E.praetermissa, Gynura miniata, Senecio abyssinicus, S.baberka, $S$. hochstetteri and $S$. lelyi. This revealed that their occurrence is sporadic. This result agrees with the findings of Gill et al., (1984) and Gill and Idu (2001) concerning the occurrence of ergastic substances in different angiospermic families.

\section{Life Form and Nature of Ergastic Substance}

Several workers have established a relationship between the life form and the nature of ergastic substances. Gill et al., (1980, 1984, 1991), Maheswari and Chakrabaty (1967) had earlier drawn a relationship between the presence of starch grains and the herbaceous habit. According to Hutchinson (1969), the herbaceous habit is more advanced than the shrubs and trees. In this family, majority of the species studied were herbs and there were no starch grain in any of the species studied.

There was no correlation between the habit of the plants with the occurrence of ergastic substances in this family. Starch grains are generally associated with herbaceous species. Interestingly, the result from this study agrees with the reports of Omoigui and Gill (1988), Gill and Abili (1989) and Idu and Gill (1998) which suggests that in the Asteraceae the presence of ergastic substances are sporadic and cannot be used in the delimitation of the taxa into genera or tribe. Thus, this characteristic nature of ergastic substances does not support these change of names as all the plants have fats and oils only. The results further support Drury and Watson (1965) and Ayodele and Olorode (2005) who cautioned on the use of limited characters for any reclassification. 
Table 1: Summary of the Locality, Habit and Nature of Ergastic Substances in the Seeds of Some Nigerian Asteraceae

\begin{tabular}{|c|c|c|c|c|c|c|}
\hline $\begin{array}{l}\text { Taxon } \\
(1)\end{array}$ & Locality (2) & $\begin{array}{l}\text { Voucher } \\
\text { Number (3) }\end{array}$ & $\begin{array}{l}\text { Habit } \\
\text { (2) }\end{array}$ & $\begin{array}{l}\text { Starch } \\
\text { (3) }\end{array}$ & $\begin{array}{l}\text { Protein } \\
\text { (4) }\end{array}$ & $\begin{array}{l}\text { Fat and } \\
\text { Oils (5) }\end{array}$ \\
\hline $\begin{array}{l}\text { TRIBE } \\
\text { Asteraceae } \\
\text { Ceruana pratensis Forsk. }\end{array}$ & $\begin{array}{l}\text { Bank of River Kaduna, } \\
\text { Kaduna, Kaduna State. }\end{array}$ & Omoigui 168 & $\mathbf{H}$ & - & + & + \\
\hline Conyza aegyptiaca (L.) Ait. var. aegyptiaca Hort. & $\begin{array}{l}\text { General Hospital, Wawa } \\
\text { Mambilla Plateau, Taraba State. }\end{array}$ & 070 & $\mathbf{H}$ & - & - & + \\
\hline C. attenuata DC. & Market Road, Amana, Cross River State, & 211 & $\mathbf{H}$ & - & - & + \\
\hline C. pyrrhopappa Sch. Bip. ex A Rich. & $\begin{array}{l}\text { Bauchi Road, by Motor Park, Jos, Plateau } \\
\text { State. }\end{array}$ & 201 & $\mathbf{H}$ & - & - & + \\
\hline C. sumatrensis (Retz.) Walker. & Ikwette Plateau, Obudu, Cross River State. & 204 & $\mathbf{H}$ & - & - & + \\
\hline Dichrocephala intergrifolia (Linn. F). O. Ktze & $\begin{array}{l}\text { Police Station, Gembu, Mambilla Plateau, } \\
\text { Taraba State }\end{array}$ & 203 & $\mathbf{H}$ & - & - & + \\
\hline $\begin{array}{l}\text { Felicia boehmii O. Hoffm. subsp. homochroma (S. } \\
\text { Moore) Grau. }\end{array}$ & Bauchi Ring Road, Jos, Plateau State. & 205 & $\mathbf{H}$ & - & - & + \\
\hline $\begin{array}{l}\text { Microglossa afzelii O. Hoffm. } \\
\text { afzelii C.D. Adams. }\end{array}$ & School of Forestry, Jos, Plateau State. & 252 & $\mathbf{H}$ & - & + & + \\
\hline $\begin{array}{l}\text { TRIBE } \\
\text { Cichorieae } \\
\text { Lactuca capensis Thumb. }\end{array}$ & Obudu Cattle Ranch, Cross River State. & 024 & $\mathbf{H}$ & - & + & + \\
\hline Launaea nana (Bak). Chiov. & $\begin{array}{l}\text { Government Rest House, Igbetti, Oyo } \\
\text { State. }\end{array}$ & 301 & $\mathbf{H}$ & - & + & + \\
\hline L. rarifolia, (Oliv. \& Hiern) L. Bolous) & Bauchi Ring Road, Jos, Plateau State. & 303 & $\mathbf{H}$ & - & + & + \\
\hline Picris xylopoda Lack. & $\begin{array}{l}\text { Lekitaba - Gembu Road, Gembu, Mambilla } \\
\text { Plateau, Taraba State. }\end{array}$ & 253 & $\mathbf{H}$ & - & + & + \\
\hline Sonchus oleraceus Linn. & Gangere Road, Kaurra Falls, Plateau State. & 256 & $\mathbf{H}$ & - & + & + \\
\hline $\begin{array}{l}\text { TRIBE } \\
\text { Cynareae }\end{array}$ & & & & & & \\
\hline Centaurea nigerica Hutch. & $\begin{array}{l}\text { Government Rest House, Igbetti, Oyo } \\
\text { State. }\end{array}$ & 133 & $\mathbf{H}$ & - & - & + \\
\hline C. perrottetii DC. & $\begin{array}{l}\text { Hadeija River Basin Authority, Kaduna Kano } \\
\text { Road, Kaduna State. }\end{array}$ & 170 & $\mathbf{H}$ & - & - & + \\
\hline C. praecox Oliv \& Hiern. & Bauchi, Ring Road, Jos, Plateau State. & 104 & $\mathbf{H}$ & - & - & + \\
\hline $\begin{array}{l}\text { Echinops giganteus A. Rich. var. lelyi (C. D. Adams) } \\
\text { C.D. Adams. }\end{array}$ & $\begin{array}{l}\text { Lekitaba Gembu Road, Gembu } \\
\text { Mambilla Plateau, Taraba State. }\end{array}$ & 035 & $\mathbf{H}$ & - & - & + \\
\hline E. gracilis $\mathrm{O}$.Hoffm. & Bauchi Ring Road, Jos, Plateau State. & 039 & $\mathbf{H}$ & - & - & + \\
\hline E. longifolius A. Rich. & Gangere Road, Kaurra Falls, Plateau State. & 038 & $\mathbf{H}$ & - & - & + \\
\hline
\end{tabular}




\begin{tabular}{|c|c|c|c|c|c|c|}
\hline \multicolumn{7}{|l|}{$\begin{array}{l}\text { TRIBE } \\
\text { Eupatorieae }\end{array}$} \\
\hline Adenostemma caffrum DC. & Bank of River Kaduna, Kaduna State. & 040 & $\mathbf{H}$ & - & - & + \\
\hline Adenostemma mauritianum DC. & $\begin{array}{l}\text { Obudu Cattle Ranch, Obudu, Cross River } \\
\text { State. }\end{array}$ & 045 & $\mathbf{H}$ & - & - & + \\
\hline A. perrottetii DC. & Bauchi Ring Road, Jos, Plateau State. & 046 & $\mathbf{H}$ & - & - & + \\
\hline Ageratum conyzoides L. & University of Jos Campus, Plateau State. & 217 & $\mathbf{H}$ & - & - & + \\
\hline Chromoleana odorata (L) King \& Robinson. & Ikwette Plateau, Obudu Cross River State. & 136 & $\mathbf{H}$ & - & - & + \\
\hline Mikania chenopodiifolia Willd. & Senior Staff Quarters Unijos, Plateau State. & 219 & $\mathbf{H}$ & - & - & + \\
\hline \multicolumn{7}{|l|}{$\begin{array}{l}\text { TRIBE } \\
\text { Heliantheae }\end{array}$} \\
\hline Acathospermum australe (Loefl.) O. Kuntze. & Bauchi Road, Jos, Plateau State. & 137 & $\mathbf{H}$ & - & - & + \\
\hline A. hispidum DC. & University of Benin, Benin City, Edo State. & 200 & $\mathbf{H}$ & - & - & + \\
\hline $\begin{array}{l}\text { Aspilia africana (Pers.) C. D. Adams var africana C.D } \\
\text { Adams. }\end{array}$ & Bauchi Ring Road, Jos, Plateau State. & 234 & $\mathbf{H}$ & - & - & + \\
\hline $\begin{array}{l}\text { A. helianthoides (Schum.\&Thonn.) Oliv. \& Hiern. } \\
\text { subsp. helianthoides O. Hoffm. }\end{array}$ & General Hospital, Igboho, Oyo State. & 202 & $\mathbf{H}$ & - & - & + \\
\hline $\begin{array}{l}\text { A. helianthoides subp. papposa (O. Hoffm. \& Muschl.) } \\
\text { C. D. Adams. }\end{array}$ & Gangere Road, Kaurra Falls, Plateau State. & 203 & $\mathbf{H}$ & - & - & + \\
\hline A. kotschyi ( Sch. Bip) Oliv. var kotschyi O. Hoffm. & A.T.B. University, Bauchi, Bauchi State. & 195 & $\mathbf{H}$ & - & - & + \\
\hline Bidens biternata (Lourn.) Merril \& Sherff. & School of Forestry, Jos, Plateau State. & 286 & & & & \\
\hline B. pilosa L. & School of Forestry, Jos, Plateau State. & 157 & $\mathbf{H}$ & - & - & + \\
\hline Coreopsis asperata Hutch. \& Dalz. & Bauchi Ring Road, Jos, Plateau State. & 157 & $\mathbf{H}$ & - & - & + \\
\hline C. bateri Oliv. \& Hiern. & Motor Park, Barakin Ladi, Plateau State. & 158 & $\mathbf{H}$ & - & - & + \\
\hline C. camporum Hutch. & Bauchi Ring Road, Jos, Plateau State. & 119 & $\mathbf{H}$ & - & - & + \\
\hline Eclipta alba (L.) Hassk. & $\begin{array}{l}\text { Ahmadu Bello University, Zaria, Kaduna } \\
\text { State }\end{array}$ & 144 & $\mathbf{H}$ & - & - & + \\
\hline Guizotia abyssinica (L.) Cass. & $\begin{array}{l}\text { Texaco Petrol Station, Lafia, Nassarawa } \\
\text { State }\end{array}$ & 193 & $\mathbf{H}$ & - & - & + \\
\hline G. scabra (Vis) Chiov. & Obudu Cattle Ranch, Cross River State & 192 & $\mathbf{H}$ & - & - & + \\
\hline Melanthera elliptica O. Hoffm. & School of Forestry, Jos, Plateau State & 126 & $\mathbf{H}$ & - & - & + \\
\hline Piloselloides hirsuta (Forssk.) C. Jeffrey. & Ikwette Plateau, Obudu, Cross River State & 118 & $\mathbf{H}$ & - & - & + \\
\hline Scleroacarpus africanus Jacq. & $\begin{array}{l}\text { Lere Gembu Road, Gembu, Mambilla } \\
\text { Plateau, Taraba State }\end{array}$ & 194 & $\mathbf{H}$ & - & - & + \\
\hline Synedrella nodiflora Gaertn. & School of Forestry, Jos Plateau State & 191 & $\mathbf{H}$ & - & + & + \\
\hline Tridax procumbens L. & Bayero University, Kano, Kano State & 010 & $\mathbf{H}$ & - & - & + \\
\hline Wedelia trilobata (L.) Hiitche. & Agodi Gate, Ibadan, Oyo State. & 180 & $\mathbf{H}$ & - & + & + \\
\hline
\end{tabular}




\begin{tabular}{|c|c|c|c|c|c|c|}
\hline $\begin{array}{l}\text { TRIBE } \\
\text { Inuleae }\end{array}$ & & & & & & \\
\hline Anisopappus africanus (Hook F.) Oliv. \& Hiern. & Ikwette Plateau, Obudu. Cross River State & 097 & $\mathbf{H}$ & - & + & + \\
\hline Anisopappus dalzelii Hutch. & Bauchi Ring Road, Jos Plateau State & 093 & $\mathbf{H}$ & - & - & + \\
\hline Gnaphalium indicum L. & Bank of River Kaduna, Kaduna State & 178 & $\mathbf{H}$ & - & - & + \\
\hline Helichrysum albiflorum Moeser. & Ikwette Plateau, Obudu Cross River State. & 071 & $\mathbf{H}$ & - & - & + \\
\hline H. foetidum (Linn.) Moench. & $\begin{array}{l}\text { Lekitaba-Gembu Road, Mambilla Plateau, } \\
\text { Taraba State }\end{array}$ & 251 & $\mathbf{H}$ & - & + & + \\
\hline H. globosum Sch. Bip. ex A. Rich. & Ikwette Plateau, Obudu Cross River State. & 252 & $\mathbf{H}$ & - & + & + \\
\hline H. mechowianum Klatt. & Ikwette Plateau, Obudu Cross River State & 254 & $\mathbf{H}$ & - & + & + \\
\hline H. quartinianum Bak. & $\begin{array}{l}\text { Wawa-Gembu Road, Gembu Mambilla } \\
\text { Plateau, Taraba State. }\end{array}$ & 255 & $\mathbf{H}$ & - & + & + \\
\hline H. rhodolepis Bak. & Ikwette Plateau, Obudu Cross River State. & 173 & $\mathbf{H}$ & - & + & + \\
\hline Inula klingii O. Hoffm. & $\begin{array}{l}\text { Lekitaba-Gembu Road, Mambilla Plateau } \\
\text { Taraba State }\end{array}$ & 218 & $\mathbf{H}$ & - & + & + \\
\hline Inula subscaposa S. Moore. & Wawa-Gembu Road. Gembu Taraba State & 178 & $\mathbf{H}$ & - & + & + \\
\hline Laggera alata (G. Don) Sch. Bip. ex Oliv. var. alata. & Gangere, Kaurra Falls, Plateau State. & 211 & $\mathbf{H}$ & - & + & + \\
\hline L. aurita (Linn. F). Benth. ex C. B. Cl. & University of Jos, Plateau State. & 257 & $\mathbf{H}$ & - & + & + \\
\hline L. braunii Vatke. & Gangere, Kaurra Falls, Plateau State. & 067 & $\mathbf{H}$ & - & + & + \\
\hline L. gracilis (O. Hoffm. \& Musch1.) C. D. Adams. & Gangere, Kaurra Falls, Plateau State. & 062 & $\mathbf{H}$ & - & + & + \\
\hline Mollera angolensis O. Hoffm. & Bauchi Ring Road, Jos, Plateau State. & 124 & $\mathbf{H}$ & - & + & + \\
\hline $\begin{array}{ll}\text { Porphryostemma chevalieri } & \text { (O. Hoffm.) Hutch. \& } \\
\text { Dalz. } & \\
\end{array}$ & School of Forestry, Jos, Plateau State. & 114 & $\mathbf{H}$ & - & + & + \\
\hline Pulicaria crispa (Forsk.) Oliv. & $\begin{array}{l}\text { Ahmadu Bello University, Zaria, Kaduna } \\
\text { State. }\end{array}$ & 141 & $\mathbf{H}$ & - & + & + \\
\hline P. undulata (Linn.) C.A. Mey. & $\begin{array}{l}\text { Usman Dan Fodio University, Sokoto, } \\
\text { Sokoto State. }\end{array}$ & 073 & $\mathbf{H}$ & - & + & + \\
\hline Sphaeranthus angustifolius DC. & $\begin{array}{l}\text { Hadeja River Basin Authority, Kaduwa } \\
\text { Kano, Kano State. }\end{array}$ & 223 & $\mathbf{H}$ & - & + & + \\
\hline S. flexuosus O. Hoffm. & $\begin{array}{l}\text { Abubakar Tafawa Balewa University, } \\
\text { Yelwa, Bauchi, Bauchi State. }\end{array}$ & 081 & $\mathbf{H}$ & - & + & + \\
\hline S. senegalensis DC. & N.A.P.R.I. Shika, Kaduna State. & 171 & $\mathbf{H}$ & - & - & + \\
\hline Vicoa leptoclada (Webb.) Dandy. & Government Rest House, Igbetti Oyo State & 155 & $\mathbf{H}$ & - & - & + \\
\hline
\end{tabular}




\begin{tabular}{|c|c|c|c|c|c|c|}
\hline $\begin{array}{l}\text { TRIBE } \\
\text { Mutiseae }\end{array}$ & & & & & & \\
\hline Dicoma tomentosa Cass. & Ahmadu Bello University, Zaria, Kaduna State. & 165 & $\mathbf{H}$ & - & - & + \\
\hline \multicolumn{7}{|l|}{$\begin{array}{l}\text { TRIBE } \\
\text { Senecioneae }\end{array}$} \\
\hline $\begin{array}{l}\text { Crassocephalum gracile (Hook. F) Milne Redhead } \\
\text { ex Guinea. }\end{array}$ & Obudu Cattle Ranch, Cross River State. & 287 & $\mathbf{H}$ & - & - & + \\
\hline C. togoense C. D. Adams. & Government Rest House, Igbetti, Oyo State & 014 & $\mathbf{H}$ & - & - & + \\
\hline Emilia coccinea (Sims) G. Don. & $\begin{array}{l}\text { General Hospital, Gembu, Mambilla Plateau } \\
\text { Taraba State }\end{array}$ & 301 & $\mathbf{H}$ & - & + & + \\
\hline E. praetermissa Milne-Redhead. & School of Forestry, Jos Plateau State & 279 & $\mathbf{H}$ & - & + & + \\
\hline Gynura miniata Welw. & Bauchi Ring Road, Jos, Plateau State. & 298 & $\mathbf{H}$ & - & + & + \\
\hline Senecio abyssinicus Sch. Bip. ex-A Rich. & School of Forestry, Jos, Plateau State. & 138 & $\mathbf{H}$ & - & + & + \\
\hline S. baberka Hutch. & Gangere, Kaurra Falls, Plateau State. & 283 & $\mathbf{H}$ & - & + & + \\
\hline S. hochstetteri Sch. Bip. ex. A Rich. & Gangere, Kaurra Plateau State. & 152 & $\mathbf{H}$ & - & + & + \\
\hline S. lelyi Hutch. & Ikwette Plateau Obudu, Cross River State. & 196 & $\mathbf{H}$ & - & + & + \\
\hline S. mannii Hook F. & $\begin{array}{l}\text { Ranch Primary School, Obudu Cross River } \\
\text { State }\end{array}$ & 151 & $\mathbf{H}$ & - & + & + \\
\hline \multicolumn{7}{|l|}{$\begin{array}{l}\text { TRIBE } \\
\text { Vernonieae }\end{array}$} \\
\hline Elephantopus mollis Kunth. & Gangere, Kaurra Falls, Plateau State. & 274 & $\mathbf{H}$ & - & - & + \\
\hline E. spicatus B. Juss. ex. Aubl. & Gangere, Kaurra Falls, Plateau State. & 148 & $\mathbf{H}$ & - & - & + \\
\hline Ethulia conyzoides Linn. F. & Eleyiele Water Works, Ibadan, Oyo State & 290 & $\mathbf{H}$ & - & - & + \\
\hline Gutenbergia nigritiana (Benth.) Oliv. \& Hiern. & Gangere, Kaurra Falls, Plateau State. & 074 & $\mathbf{H}$ & - & - & + \\
\hline G. rueppellii Sch. Bip. & $\begin{array}{l}\text { Abuja-Kaduna Road, Lere Junction, Kaduna } \\
\text { State. }\end{array}$ & 027 & $\mathbf{H}$ & - & - & + \\
\hline Vernonia ambigua Kotschyi \& Peyr. & School of Forestry, Jos Plateau State. & 106 & $\mathbf{H}$ & - & - & + \\
\hline V. biafrae Oliv. \& Hiern. & School of Forestry, Jos Plateau State. & 129 & $\mathbf{S}$ & - & - & + \\
\hline V. calvoana (Hook. f.) Hook.F. var. calvoana & A.T.B University, Bauchi, Bauchi State. & 302 & $\mathbf{H}$ & - & - & + \\
\hline V. camporum A. Chev. & Ahmadu Bello University, Zaria, Kaduna State. & 306 & $\mathbf{S}$ & - & - & + \\
\hline V. cistifolia O. Hoffm. & Obudu Cattle Ranch Hotel, Cross River State. & 107 & $\mathbf{H}$ & - & - & + \\
\hline V. colorata (Willd.) Drake. & Obudu Cattle Ranch, Obudu Cross River State. & 137 & $\mathbf{H}$ & - & - & + \\
\hline V. conferta Benth. & Lekitaba-Gembu Road, Gembu, Taraba State. & 178 & $\mathbf{H}$ & - & - & + \\
\hline V. galamensis (Cass.) Less. & Government Rest House, Igbetti, Oyo State. & 245 & $\mathbf{H}$ & - & - & + \\
\hline V. gerberiformis Oliv. \& Hiern. & Ahmadu Bello University, Zaria, Kaduna State. & 120 & $\mathbf{H}$ & - & - & + \\
\hline V. glaberimma Welw ex O. Hoffm. & School of Forestry, Jos, Plateau State. & 116 & $\mathbf{H}$ & - & - & + \\
\hline V. guineensis Benth. var. cameroonica C.D. Adams. & $\begin{array}{l}\text { Market Road, Amana, Obudu, Cross River } \\
\text { State. }\end{array}$ & 108 & $\mathbf{S}$ & - & - & + \\
\hline
\end{tabular}


Bajopas Volume 5 Number 2 December, 2012

\begin{tabular}{|c|c|c|c|c|c|c|}
\hline V. guineensis Benth. var. guineensis V. Firma. & Bauchi Ring Road, Jos, Plateau State. & 240 & H & - & - & + \\
\hline V. iodocalyx O. Hoffm. & Obudu Cattle Ranch, Cross River State. & 242 & $\mathbf{H}$ & - & - & + \\
\hline V. ituriensis Muschl. var. occidenta/C. Jeffrey. & School of Forestry, Jos, Plateau State. & 105 & $\mathbf{H}$ & - & - & + \\
\hline V. nestor S. Moore. & Gangere Road Kaurra Falls, Plateau State. & 135 & $\mathbf{S}$ & - & - & + \\
\hline V. nigritiana Oliv. \& Hiern. & Bauchi Ring Road, Jos, Plateau State. & 131 & $\mathbf{H}$ & - & - & + \\
\hline V. perrottetii Sch. Bip. & Gangere Road, Kaurra Falls, Plateau State. & 130 & $\mathbf{H}$ & - & - & + \\
\hline V. philipsoniana (Lawalree) & Bauchi Ring Road, Jos, Plateau State. & 227 & $\mathbf{H}$ & - & - & + \\
\hline V. richardiana (O. Ktze) P. Sermolli. & Jekko Road, Kaurra Falls, Plateau State. & 126 & $\mathbf{H}$ & - & - & + \\
\hline V. saussureoides Hutch. & Gangere Road, Kaurra Falls, Plateau State. & 092 & $\mathbf{S}$ & - & - & + \\
\hline V. smithiana Less. & $\begin{array}{l}\text { Lekitaba Gembu Road, Mambilla Plateau } \\
\text { Taraba State. }\end{array}$ & 281 & $\mathbf{H}$ & - & - & + \\
\hline V. stenocephala Oliv. & Bauchi Ring Road, Jos, Plateau State. & 109 & $\mathbf{S}$ & - & - & + \\
\hline V. stenostegia (Stap F.) Hutch. \& Dalz. & Bauchi Road, Unijos, Jos, Plateau State. & 093 & $\mathbf{H}$ & - & - & + \\
\hline V. subaphylla Bak. & Ikwette Plateau, Obudu, Cross River State. & 143 & $\mathbf{H}$ & - & - & + \\
\hline V. tenoreana Oliv. & General Hospital, Igboho, Oyo State. & 042 & $\mathbf{H}$ & - & - & + \\
\hline
\end{tabular}

KEY: H: Herb, S: Shrub, +: Present, - : Absent 


\section{REFERENCES}

Adams, C.D. (1963). Compositae. In: Hutchinson, J and J.M. Dalziel. Flora of West Tropical Africa. $2^{\text {nd }}$ Ed. (F.M. Hepperd Ed.). Crown Agents, London. Pp. 271-283.

Ayodele, M. S. and Olorode, O. (2005). A biosystematic evaluation of the relationship between three allopathic shrubby species of Vernonia Schreb (Asteraceae) in Nigeria. Compositae Newsletter, 42:8-25..

Drury, D. G. and Watson, L. (1965). Anatomy and the taxonomic significance of gross vegetative morphology in Senecio. New Phytologists 64: 307-314.

Erdtman, H. (1962). Flavinoid heartwood constituents of Conifers. Science Proceedings of Botany 16: 221-250.

Funk, V.A. (2010). Ten things I learned on the way to the mother tree (i.e. mother ship). Compositae Newsletter 48: 6-21.

Gill, L.S. and Abili, M.A. (1989). Ergastic substances in seeds. V. Feddes Repertorium 100: 7179.

Gill, L.S. and Ayodele, J.R. (1986). On the nature of ergastic substances in the seeds of some tropical and temperate angiosperms III. Journal of Plant Anatomy and Morphology 3: 35-37.

Gill, L.S. and Idu, M. (2001). On the nature of ergastic substances in some Caryophyllaceae seeds-IX. Nigerian Journal of Applied Science 19: 10-16.

Gill, L.S., Nyawuame, H.G.K., Aibangbe, M.I. and Agho, D.A. (1991). Nature of ergastic substances in some Mediterranean angiospermous seeds. VI. Feddes Repertorium 102: 613-628.

Gill, L.S., Olabanji, G.O. and Husaini, S.W.H. (1980). On the nature of stored food material in the seeds of some Nigerian Legumes. Legume Research 3: 67-70.

Gill, L.S., Olabanji, G.O. and Husaini, S.W.H. (1984). On the nature of stored food material in the seeds of some Nigerian Leguminosae II. Feddes Repertorium 95: 659-663.

Hutchinson, J. (1969). Evolution and Phylogeny of Flowering Plants. Academic Press, London. 717p.

Idu, M. and Gill, L.S. (1998). Nature of ergastic substances in some West African Asteraceae. Compositae Newsletter 32: 5056.

Isawumi, M. A. (1993). New combinations in Baccharoides Monech (VernonieaeCompositae) in West Africa, Feddes Repertorium 104: 309-326.
Maheswari, J.K. and Chakrabarty (1967). Starch grains in leguminous seeds. Phyton (Austrian) 12: 1-24.

Omoigui, I.D. and Gill, L.S. (1988). Nature of ergastic substances in some West African Compositae. Feddes Repertorium 99:143145.

Robinson, H. (1990). Six new combinations in Bacchariodes Moench and Cyanthillium Blume (Vernonieae: Asteraceae). Proceeding of Biological Society, Washington 103: 248253. 